\title{
A new class of graphs that satisfies the Chen-Chvátal Conjecture*
}

\author{
P. Aboulker ${ }^{a}$, M. Matamala ${ }^{b, c}$, P. Rochet ${ }^{c, d}$ and J. Zamora ${ }^{e}$ \\ (a) Project Coati, I3S (CNRS, UNSA) and INRIA, Sophia Antipolis, France \\ (b) Depto. Ingeniería Matemática (DIM), Universidad de Chile \\ (c) Centro de Modelamiento Matemático (CMM, UMI 2807 CNRS), Universidad de Chile \\ (d) Laboratoire de Mathématiques Jean Leray, Université de Nantes \\ (e) Depto. Matemáticas, Universidad Andres Bello
}

October 4, 2018

\begin{abstract}
A well-known combinatorial theorem says that a set of $n$ non-collinear points in the plane determines at least $n$ distinct lines. Chen and Chvátal conjectured that this theorem extends to metric spaces, with an appropriated definition of line. In this work we prove a slightly stronger version of Chen and Chvátal conjecture for a family of graphs containing chordal graphs and distance-hereditary graphs.
\end{abstract}

\section{Introduction}

A classic result in Euclidean geometry asserts that every non-collinear set of $n$ points in the Euclidean plane determines at least $n$ distinct lines.

Erdös [14] showed that this result is a consequence of the Sylvester-Gallai theorem which asserts that every non-collinear set of $n$ points in the plane determines a line containing precisely two points. Coxeter [11] showed that the Sylvester-Gallai theorem holds in a more basic setting known as ordered geometry. Here, the notions of distance and angle are not used and, instead, a ternary relation of betweenness is employed. We write $[a b c]$ for the statement that $b$ lies between $a$ and $c$. In this notation, a line $\overline{x y}$ is defined (for any two distinct points $x$ and $y$ ) as:

$$
\overline{x y}=\{x, y\} \cup\{u:[u x y] \text { or }[x u y] \text { or }[x y u]\}
$$

Betweenness in metric spaces was first studied by Menger [16] and further on by Chvátal [9]. In a metric space $(V, d)$, we define

$$
[a b c] \Leftrightarrow d(a, b)+d(b, c)=d(a, c) .
$$

Hence, in any metric space $(V, d)$, we can define the line $\overline{u v}$ induced by two points $u$ and $v$ as in (1). A line of a metric space $(V, d)$ is universal if it contains all points of $V$. With this definition of lines in metric spaces, Chen and Chvátal [6] proposed the following beautiful conjecture.

Conjecture 1.1 Every metric space on $n$ points, where $n \geq 2$, either has at least $n$ distinct lines or has a universal line.

${ }^{*}$ Partially supported by Basal program PBF 03 and Núcleo Milenio Información y Coordinación en Redes ICM/FIC P10-024F. 
The best known lower bound for the number of lines in metric spaces with no universal line is $\Omega(\sqrt{n})[2$.

As it is explained in 3, it suffices to prove Conjecture 1.1 for metric spaces with integral distances. This motivates looking at two particular types of metric spaces. First, for a positive integer $k$, we define a $k$-metric space to be a metric space in which all distances are integral and are at most $k$. Chvátal [10] proved that every 2-metric space on $n$ points $(n \geq 2)$ either has at least $n$ distinct lines or has a universal line. The question is open for $k \geq 3$. Aboulker et al. [2] proved that, for all $k \geq 3$, a $k$-metric space with no universal line has at least $n / 5 k$ distinct lines.

A second type of metric space with integral distances arises from graphs. Any finite connected graph induces a metric space on its vertex set, where the distance between two vertices $u$ and $v$ is defined as the length of a shortest path linking $u$ and $v$. Such metric spaces are called graph metrics and are the subject of this paper. The best known lower bound on the number of lines in a graph metric with no universal line is $\Omega\left(n^{4 / 7}\right)[2]$. In [5] and [3] it is proved that Conjecture 1.1 holds for chordal graphs and for distance-hereditary graphs respectively. The main result of this paper is to prove Conjecture 1.1 for all graphs that can be constructed from chordal graphs by repeated substitutions and gluing along vertices. This generalizes chordal and distance hereditary graphs.

\section{Statement of the main theorem}

Let $G=(V, E)$ be a connected graph. Let $a, b, c$ be three distinct vertices in $V(G)$. The distance $d_{G}(a, b)$ (or simply $d(a, b)$ when the context is clear) between $a$ and $b$ is the length of a shortest path linking $a$ and $b$. We write $[a b c]^{G}$ (or simply $[a b c]$ ) when $d(a, b)+d(b, c)=d(a, c)<\infty$. Observe that $[a b c] \Leftrightarrow[c b a]$. We denote by $\overline{a b}^{G}$ (or simply $\overline{a b}$ ) the line induced by two distinct vertices $a, b$. Recall that $\overline{a b}^{G}=\{a, b\} \cup\{x:[a b x]$ or $[a x b]$ or $[x a b]\}$. Notice that with this definition the line defined by two vertices $a, b$ lying in different connected components is $\{a, b\}$. We denote by $\mathcal{L}(G)$ the set of distinct lines in $G$ and by $\ell(G)=|\mathcal{L}(G)|$ the number of distinct lines in $G$.

We denote by $N_{G}(v)$ the set of all neighbors of a vertex $v$ in $G$. For a set of vertices $S$, we denote by $N_{G}(S)$ (or simply $N(S)$ ) the set of all vertices outside $S$ having a neighbor in $S$. A set $S$ is dominating if $S \cup N_{G}(S)=V(G)$.

A set of vertices $M$ of a graph $G=(V, E)$ is a module if for each $a, b \in M, u \notin M, a u \in E$ if and only if $b u \in E$. It is a non-trivial module if $|V|>|M| \geq 2$. If $M$ is a dominating set, we call it a dominating module. In this situation, $N(M)$ is also a module unless $M=V$. When $M=\{u, v\}$, we say that $(u, v)$ is a pair of twins. If $u$ and $v$ are adjacent they are called true twins; otherwise, they are called false twins. A graph without non-trivial module is called a prime graph.

A bridge $a b$ is an edge whose deletion disconnects the graph. We denote by $\operatorname{br}(G)$ the number of bridges of $G$. If $\operatorname{br}(G)=0$, we say that $G$ is bridgeless. If $a b$ is a bridge of a graph $G$, then for every vertex $p \in V(G) \backslash\{a, b\}$, we either have $[p a b]$ or $[a b p]$. Hence $\overline{a b}^{G}=V(G)$ and thus Conjecture 1.1 is only interesting for bridgeless graphs.

Let $\mathcal{C}$ be the class of graphs $G$ such that every induced subgraph of $G$ is either a chordal graph, has a cut-vertex or a non-trivial module. By definition, this class is hereditary, that is, if $G \in \mathcal{C}$, then every induced subgraph of $G$ is also in $\mathcal{C}$.

Let $\mathcal{F}=\left\{C_{4}, K_{2,3}, W_{4}, W_{4}^{\prime}, K_{6}^{\prime}, K_{8}^{\prime}\right\}$ (see Figure 1). In this work we prove the following theorem.

Theorem 2.1 For each connected graph $G \in \mathcal{C} \backslash \mathcal{F}, \ell(G)+\operatorname{br}(G) \geq|G|$. 

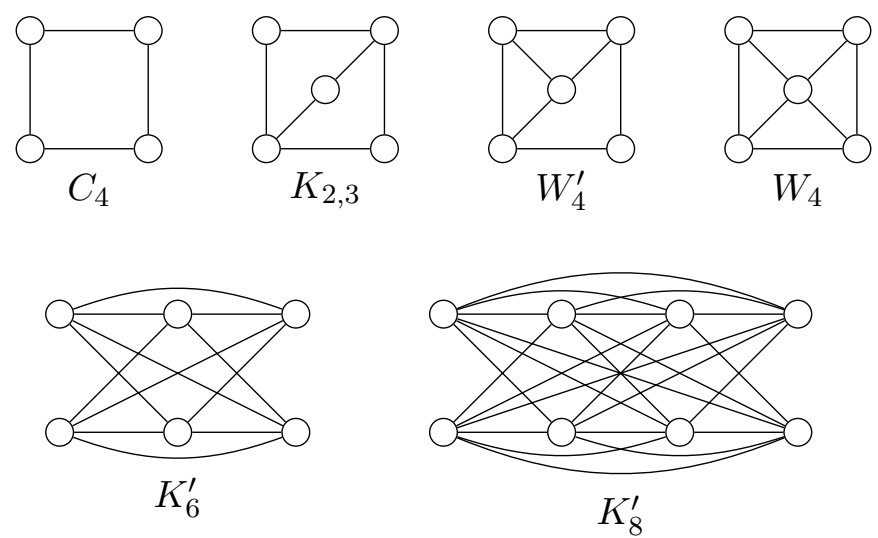

Figure 1: Graphs in $\mathcal{F}$.

As a consequence we have that Chen-Chvatal conjecture holds for $\mathcal{C}$, as it holds for graphs in $\mathcal{F}$ (they all have a universal line). Since all distance-hereditary graphs contain either a pendant edge or a pair of twins, $\mathcal{C}$ is a super class of distance hereditary graphs. It is also clearly a super class of chordal graphs.

The difference between our result and the original conjecture is that we count a universal line as any other line but, since each bridge defines a universal line, we count it with multiplicity. It is tempting to conjecture that the property $\ell(G)+b r(G) \geq|G|$ holds for all graphs but a finite number. We know this to be false, as it was pointed out to us by Yori Zwols, owing to a simple observation: a counter-example containing a bridge produces an infinite number of counter-examples from replacing a bridge by a path of arbitrary length. So far, the three known minimal counter-examples containing a bridge are shown in Figure 2, It remains unknown however whether all counter-examples to $\ell(G)+b r(G) \geq|G|$ can be obtained from a finite set of graphs upon replacing a bridge by a path.
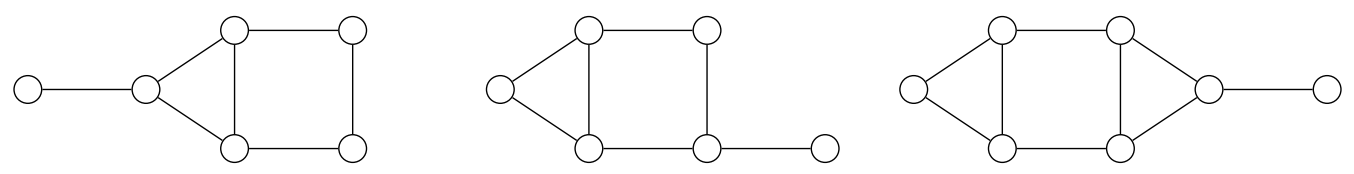

Figure 2: The three known minimal counter-examples with a bridge to $\ell(G)+b r(G) \geq|G|$.

Since for these three graphs, the bridge is a pendant edge, we venture to propose the following conjecture.

Conjecture 2.2 There is a finite set of graphs $\mathcal{F}_{0}$ such that every connected graph $G \notin \mathcal{F}_{0}$ either has a pendant edge or satisfies $\ell(G)+b r(G) \geq|G|$.

So far, we know that if such a family $\mathcal{F}_{0}$ exists, it contains the list of graphs in Figure 1 and Figure 2. An interesting variation of the conjecture can be stated as follows, denoting by $u l(G)$ the number of pairs of vertices in $G$ that induce a universal line.

Conjecture 2.3 For every connected graph $G, \ell(G)+u l(G) \geq|G|$. 
Although less general (a bridge always induces a universal line but not all universal lines are induced by bridges), this conjecture has the merit of being true for all the known graphs in $\mathcal{F}$. Thus, there is no known counter-examples to Conjecture 2.3 to this day. Moreover, it remains stronger than the original Chen-Chvatal conjecture without ruling out graphs with universal lines as trivial solutions.<smiles>O1OOO1</smiles><smiles>C1OOC2(O1)OOC21OCOO1</smiles>

$H_{8}^{1}$
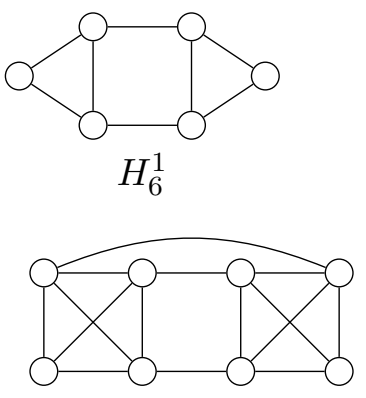

$H_{8}^{2}$
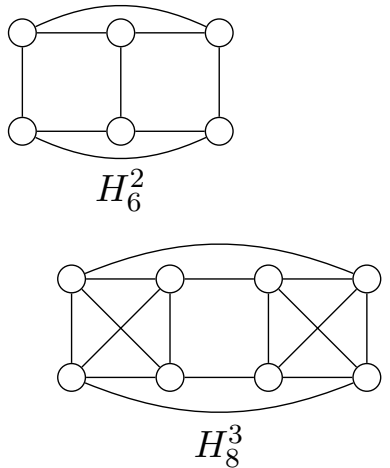

Figure 3: Known graphs in $\mathcal{F}_{0} \backslash \mathcal{F}$.

\section{Preliminaries}

In this section, we give some results on the number of lines of graphs in $\mathcal{F}$ or that are constructed from a graph in $\mathcal{F}$ adding a vertex. The proof of the following Lemma is done by brute force using a computer 1 . (See Figure 4.)

Lemma 3.1 $\ell\left(C_{4}\right)=1, \ell\left(K_{6}^{\prime}\right)=4$ and for $H \in \mathcal{F} \backslash\left\{C_{4}, K_{6}^{\prime}\right\}, \ell(H)=|H|-1$.

Lemma 3.2 Let $G \in \mathcal{C} \backslash \mathcal{F}$ be a graph.

1) If $G$ has a pendant vertex $v$ such that $G-v \in \mathcal{F} \backslash\left\{C_{4}\right\}$, then $\ell(G)+\operatorname{br}(G)=\ell(G)+1 \geq|G|$.

2) If $G$ contains a non-trivial module $M$ and $G-v \in \mathcal{F} \backslash\left\{C_{4}\right\}$, for some $v \in M$, then $\ell(G) \geq|G|+1$.

Proof : The proofs of both statements are easy although tedious. In the first case, if $u$ is the neighbor of $v$ in $G$, then $\overline{w v}^{G}$ defines different lines, when $w$ varies over the neighbors of $u$ in $G-v$. These lines are not in $\mathcal{L}(G-v)$ if $G-v \in \mathcal{F} \backslash\left\{C_{4}\right\}$. Since the graphs in $\mathcal{F}$ have no vertex of degree one, we obtain at least two new lines.

In the second case, for each $G^{\prime} \in \mathcal{F}$ we need to consider all graphs $G$ arising from $G^{\prime}$ by adding a copy $v$ of a vertex $v^{\prime}$ in $G^{\prime}$ so as $\left(v, v^{\prime}\right)$ is a pair of twins (true or false) in $G$. We do this with the help of a computer program ${ }^{2}$.

\footnotetext{
${ }^{1}$ The details can be found http://www.math.sciences.univ-nantes.fr/ rochet/recherche/Lines_in_F pdf.

For the sake of completeness, the $\mathrm{R}$ code and environment used to check all the cases are available in http: //www.math.sciences.univ-nantes.fr/ rochet/recherche/Code_lines.R and in http://www.math.sciences univ-nantes.fr/ rochet/recherche/env_lines.RData.
} 


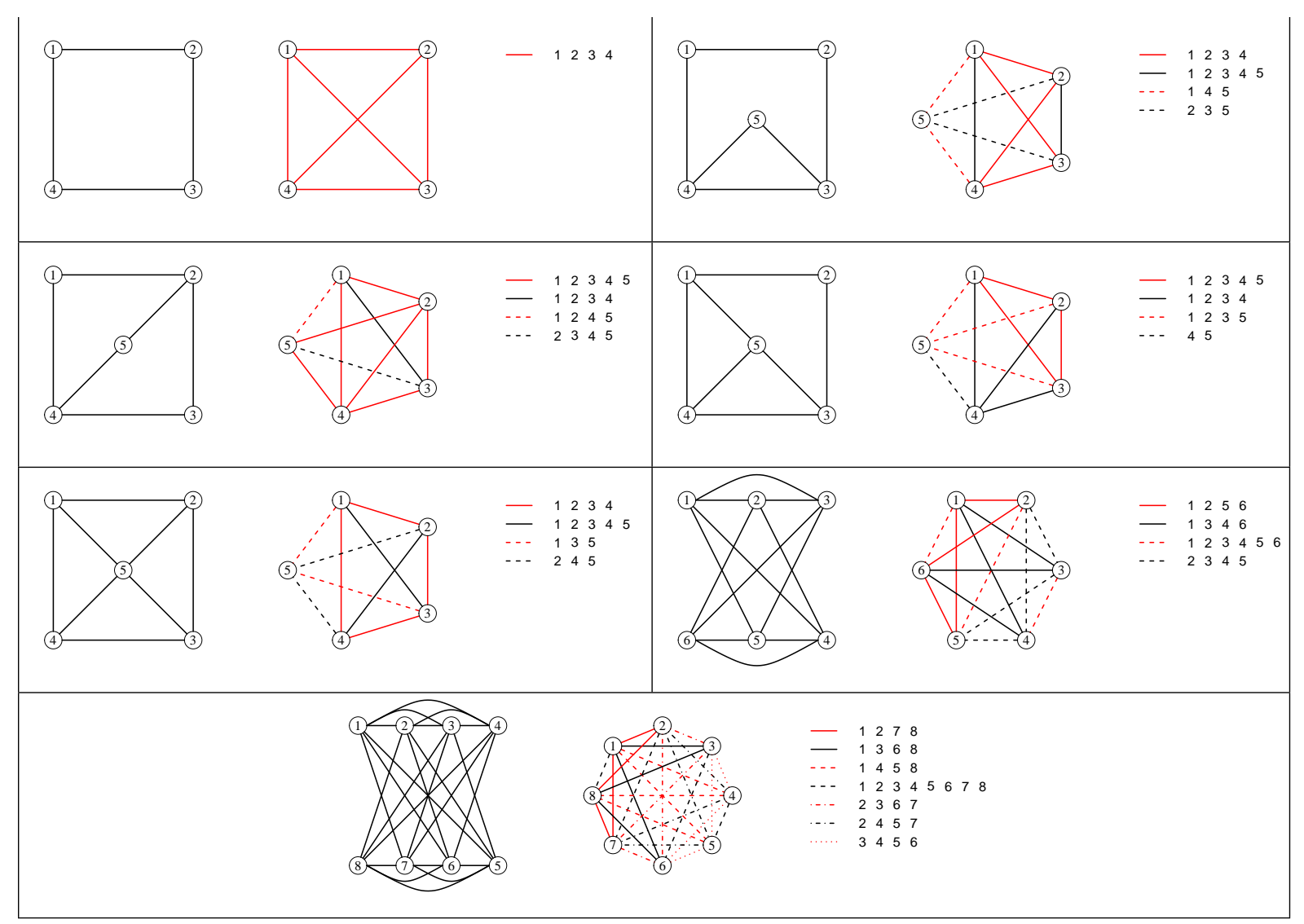

Figure 4: Members of $\mathcal{F}$. For each graph in $\mathcal{F}$, three drawings appear. To the left, the graph itself. In the middle, an edge-colored complete graph where pairs of vertices defining the same line have the same color. To the right, the set of vertices in each line (color). 


\section{Proof of Theorem 2.1}

We prove Theorem 2.1 by induction on the number of vertices of $G$. Let $G \in \mathcal{C} \backslash \mathcal{F}$ with $|G|=n$. The proof splits in four parts: (1) $G$ has a bridge, (2) $G$ has no bridge and has a cut-vertex, (3) $G$ is 2-connected and chordal, (4) $G$ is 2-connected and has a non trivial module.

\section{Part 1: $G$ has a bridge.}

Let $u_{1} u_{2}$ be a bridge of $G$. Let $G_{1}$ and $G_{2}$ be the connected components of $G-u_{1} u_{2}$ that contains respectively $u_{1}$ and $u_{2}$. To contract an edge $e$ of a graph $G$ is to delete $e$ and then identify its ends. Let $G^{\prime}$ be the graph obtained from $G$ by contracting $u_{1} u_{2}$. Name $u$ the vertex of $G^{\prime}$ appeared after the contraction. The following claim which easy proof is omitted, say that for any two vertices in $G^{\prime}-\{u\}$ the lines $\overline{x y}^{G}$ and $\overline{x y} \bar{G}^{\prime}$ might differ only in $\left\{u, u_{1}, u_{2}\right\}$.

$$
\overline{x y}^{G}= \begin{cases}\overline{x y} G^{G^{\prime}} & \text { if } u \notin \overline{x y}^{G^{\prime}} \\
\overline{x y} G^{\prime}-\{u\} \cup\left\{u_{i}\right\} & \text { if } x, y \in V\left(G_{i}\right),[x u y]^{G^{\prime}} \text { and } i \in\{1,2\} \\
\overline{x y} G^{G^{\prime}}-\{u\} \cup\left\{u_{1}, u_{2}\right\} & \left\{\begin{array}{l}
\text { if } x, y \in V\left(G_{i}\right) \text { for an } i \in\{1,2\} \text { and }[x y u]^{G^{\prime}} \text { or }[y x u]^{G^{\prime}} \text { or, } \\
\text { if } x \in V\left(G_{i}\right) \text { and } y \in V\left(G_{3-i}\right) \text { for an } i \in\{1,2\}
\end{array}\right.\end{cases}
$$

And in the case that $y=u$ we have that:

$$
\overline{u x} G^{\prime}-\{u\}=\bar{u}_{1}^{G}-\left\{u_{1}, u_{2}\right\} .
$$

This implies that $\ell(G) \geq \ell\left(G^{\prime}\right)$. Moreover it is clear that $\operatorname{br}(G)=\operatorname{br}\left(G^{\prime}\right)+1$. If $G^{\prime} \in \mathcal{C} \backslash \mathcal{F}$, then by induction we have $\ell\left(G^{\prime}\right)+\operatorname{br}\left(G^{\prime}\right) \geq\left|G^{\prime}\right|=|G|-1$ and thus $\ell(G)+\operatorname{br}(G) \geq|G|$ and we are done.

So we may assume that $G^{\prime} \in \mathcal{F}$. Since graphs in $\mathcal{F}$ are 2 -connected, it implies that $u_{1} u_{2}$ is a pendant edge of $G$. Then the result follows by Lemma 3.2 when $G^{\prime} \neq C_{4}$, and it is easily checkable when $G^{\prime}=C_{4}$. This ends the Part 1 .

\section{Part 2: $G$ has no bridge and has a cut-vertex.}

Let $u$ be a cut-vertex of $G$. Let $C_{1}$ be a connected component of $G-\{u\}$ and let $C_{2}$ be the union of the other connected components of $G$. Set $G_{1}=G\left[C_{1} \cup\{u\}\right]$, and $G_{2}=G\left[C_{2} \cup\{u\}\right]$. Observe that, since $G$ is bridgeless, $G_{1}$ and $G_{2}$ are also bridgeless.

Claim (1) below, whose easy proof is omitted, implies that, for $i=1,2$, a line induced by two vertices in $V\left(G_{i}\right)$ is either disjoint from $V\left(C_{3-i}\right)$ or contains $V\left(C_{3-i}\right)$. In particular, it implies that a line induced by two vertices in $V\left(G_{1}\right)$ is distinct from a line induced by two vertices in $V\left(G_{2}\right)$, except in the perverse case where this line is universal.

(1) For $i=1,2$ and for all $x, y \in V\left(G_{i}\right)$ we have:

- if $[x y u]$ or $[y x u]$, then $\overline{x y}^{G}=\overline{x y}^{G_{i}} \cup V\left(C_{3-i}\right)$,

- otherwise $\overline{x y}^{G}=\overline{x y}^{G_{i}}$ and in particular $\overline{x y}^{G} \cap V\left(C_{3-i}\right)=\emptyset$.

We next prove the following lower bound for $\ell(G)$.

(2) $\ell(G) \geq \ell\left(G_{1}\right)+\ell\left(G_{2}\right)-1+\left|N_{G_{1}}(u)\right|\left|N_{G_{2}}(u)\right|$.

For $i=1,2$, let $\mathcal{L}_{i}=\left\{\overline{a b}^{G}: a, b \in V\left(G_{i}\right)\right\}$. By $(1)\left|\mathcal{L}_{i}\right|=\ell\left(G_{i}\right)$, and the only possible line in $\mathcal{L}_{1} \cap \mathcal{L}_{2}$ is the universal line. Hence $\left|\mathcal{L}_{1} \cup \mathcal{L}_{2}\right| \geq \ell\left(G_{1}\right)+\ell\left(G_{2}\right)-1$. Moreover, for all lines $l$ in $\mathcal{L}_{1} \cup \mathcal{L}_{2}, l$ contains either $V\left(C_{1}\right)$ or $V\left(C_{2}\right)$. 

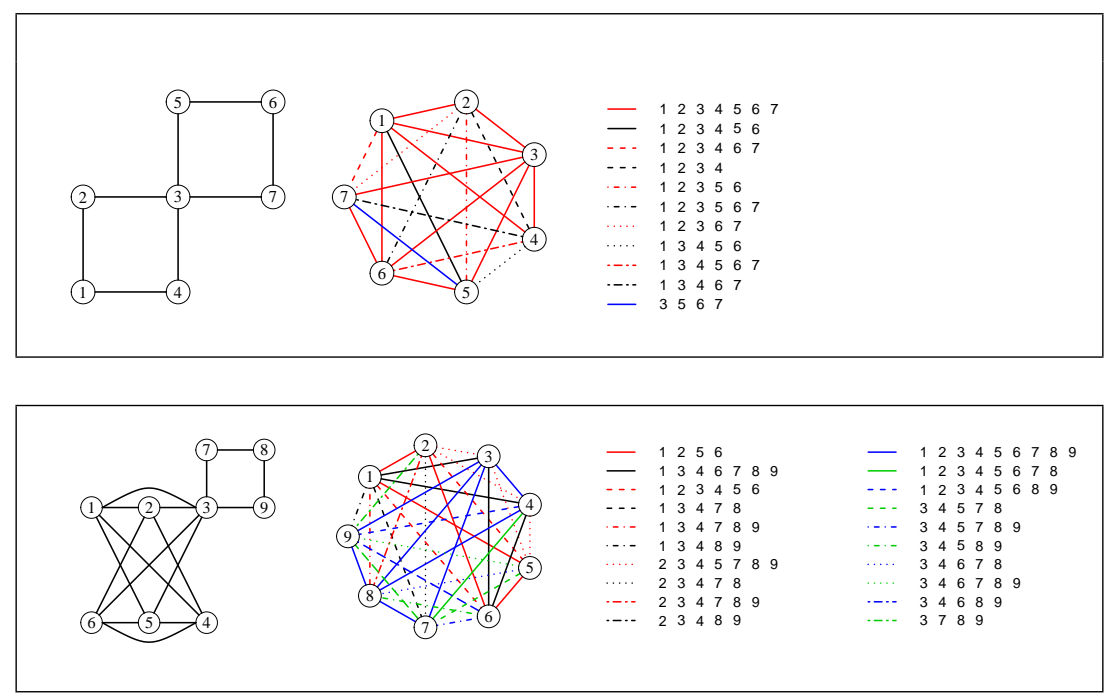

Figure 5: Lines of the graphs obtained by gluing, respectively, a cycle of length four and a $K_{6}^{\prime}$ to a $C_{4}$. To the right we represent the set of lines as an edge coloring of the complete graph, together with the elements in each set.

For $i=1,2$, let $u_{i}$ be a neighbor of $u$ in $G_{i}$. We have that $\overline{u_{1} u_{2}} G \cap N(u)=\left\{u_{1}, u_{2}\right\}$ and since $u$ has at least two neighbors in both $G_{1}$ and $G_{2}$ (because $G$ is bridgeless), then ${\overline{u_{1} u_{2}}}^{G} \cap V\left(G_{i}\right) \notin\left\{\emptyset, V\left(G_{i}\right)\right\}$ for each $i=1,2$ and thus it is distinct from all lines in $\mathcal{L}_{1} \cup \mathcal{L}_{2}$. Moreover, for every $u_{i}, v_{i}$ neighbors of $u$ in $G_{i}$, for each $i=1,2$, if $\left\{u_{1}, u_{2}\right\} \neq\left\{v_{1}, v_{2}\right\}$, then $\overline{u_{1} u_{2}} G \neq{\overline{v_{1} v_{2}}}^{G}$. Therefore, there are at least $\left|N_{G_{1}}(u)\right|\left|N_{G_{2}}(u)\right|$ lines in $\mathcal{L}(G) \backslash\left(\mathcal{L}_{1} \cup \mathcal{L}_{2}\right)$. This proves (2).

Since $\left|N_{G_{1}}(u)\right|\left|N_{G_{2}}(u)\right| \geq 4$ we get $\ell(G) \geq \ell\left(G_{1}\right)+\ell\left(G_{2}\right)+3$. If $\ell\left(G_{1}\right)+\ell\left(G_{2}\right) \geq\left|G_{1}\right|+\left|G_{2}\right|-4$, then $\ell(G) \geq\left|G_{1}\right|+\left|G_{2}\right|-1=|G|$. If $\ell\left(G_{1}\right)+\ell\left(G_{2}\right) \leq\left|G_{1}\right|+\left|G_{2}\right|-3$, by the induction hypothesis, we conclude that both $G_{1}$ and $G_{2}$ are in $\mathcal{F}$. Moreover, from Lemma 3.1 we get that $G_{1}=G_{2}=C_{4}$ or $\left\{G_{1}, G_{2}\right\}=\left\{C_{4}, K_{6}^{\prime}\right\}$. We have verified that in the first case we have 11 lines, while in the second, we have 20 lines (see Figure 5). This ends the Part 2.

\section{Part 3: $G$ is 2-connected and chordal.}

In [5] it was proved that Conjecture 1.1 holds for chordal graphs. The proof of this part is the same as their proof. We first need Lemma 1 of [5]:

Lemma 4.1 Let $G$ be a chordal graph and let $s, x, y$ in $V(G)$ such that [sxy]. If $\overline{s x}=\overline{s y}$, then $x$ is a cut-vertex of $G$.

A vertex of a graph is called simplicial if its neighbors are pairwise adjacent. By a classic result of Dirac [13, a chordal graph has at least two simplicial vertices. Let $s$ be a simplicial vertex of $G$. Since $s$ is simplicial for any pair of vertices $x, y \in V(G) \backslash\{s\},[x s y]$ does not hold. Hence, if $\overline{s x}=\overline{s y}$, we must have $[s x y]$ or $[s y x]$ and thus, by Lemma 4.1, $x$ or $y$ is a cut vertex, a contradiction.

Hence, the set $\{\overline{s u}: u \in V(G) \backslash\{s\}\}$ has $n-1$ distinct lines. Observe that all these lines contain $s$. Now, since $G$ is 2-connected, $s$ has at least two neighbors $a, b$ and $s \notin \overline{a b}$. 


\section{Part 4: $G$ is 2-connected, non-chordal and has a non trivial module}

We first consider the case when $G$ has a non-trivial non-dominating module.

Let $M=\left\{v_{1}, \ldots v_{s}\right\}$ be a non-trivial non-dominating module of $G$ with neighborhood $N(M)$ of minimal size. Set $G^{\prime}:=G-\left\{v_{1}\right\}$. If $G^{\prime} \in \mathcal{F} \backslash\left\{C_{4}\right\}$, we are done by Lemma 3.2. If $G^{\prime}=C_{4}$, then $G$ is either $K_{2,3}$ or $W_{4}^{\prime}$, a contradiction with $G \notin \mathcal{F}$. So we may assume that $G^{\prime} \notin \mathcal{F}$ and from the induction hypothesis we get $\ell\left(G^{\prime}\right)+\operatorname{br}\left(G^{\prime}\right) \geq\left|G^{\prime}\right|=|G|-1$.

Set $\mathcal{L}^{\prime}=\left\{\overline{x y}^{G}: x, y \in V\left(G^{\prime}\right)\right\}$. Since $G^{\prime}$ is an isometric subgraph of $G$ (i.e. for all $\left.x, y \in V\left(G^{\prime}\right), d_{G^{\prime}}(x, y)=d_{G}(x, y)\right)$, we have, for all $a, b \in V\left(G^{\prime}\right), \overline{a b}^{G}=\overline{a b}^{G^{\prime}}$ or $\overline{a b}^{G^{\prime}} \cup\left\{v_{1}\right\}$. Hence

$$
\left|\mathcal{L}^{\prime}\right|=\ell\left(G^{\prime}\right) \geq|G|-1-\operatorname{br}\left(G^{\prime}\right) .
$$

Moreover, each line in $\mathcal{L}^{\prime}$ that contains $v_{1}$ must contain at least one other vertex of $M$. In effect, let $\overline{a b} \in \mathcal{L}^{\prime}$ such that $v_{1} \in \overline{a b}$. If $a, b \notin M$, then $\overline{a b}$ contains $M$ and we are done, otherwise either $a$ or $b$ are in $M$ and thus $\overline{a b}$ contains at least two vertices of $M$.

Let $t \in G-(M \cup N(M))$. It is clear that $v_{1}$ is the unique vertex in $M$ which belongs to the line ${\overline{v_{1}}}^{G}$. Hence, ${\overline{v_{1} t}}^{G} \notin \mathcal{L}^{\prime}$ and thus, if $b r\left(G^{\prime}\right)=0$, we are done by $(2)$.

So we may assume that $G^{\prime}$ has at least one bridge. Let $a b$ be a bridge of $G^{\prime}$, and let $G_{a}$, $G_{b}$ be the connected components of $G^{\prime}-a b$ that contains respectively $a$ and $b$. We are going to prove that one of $G_{a}, G_{b}$ is reduced to one vertex of degree exactly 2 and that this vertex is in $N(M)$ (so it also implies that $|M|=2$ ). Since a vertex in $G_{a}$ has at most one common neighbor with a vertex in $G_{b}$ and $|N(M)| \geq 2$, because $G$ is 2-connected and thus two vertices in $M-\left\{v_{1}\right\}$ have at least two common neighbors in $G^{\prime}, M-\left\{v_{1}\right\}$ cannot intersect both $G_{a}$ and $G_{b}$. So we may assume without loss of generality that $M-\left\{v_{1}\right\} \subseteq V\left(G_{a}\right)$. Since $a b$ is not a bridge of $G, v_{1}$ must have a neighbor in both $G_{a}$ and $G_{b}$. Hence the only neighbor of $v_{1}$ in $G_{b}$ is $b, v_{2}=a$ and $M=\left\{v_{1}, v_{2}\right\}$. Moreover, since $G$ has no cut-vertex, $G_{b}=\{b\}$. Finally, by minimality of $N(M), v_{2} b$ is the unique bridge of $G^{\prime}$. Indeed, if $G^{\prime}$ has another bridge, then there exists a vertex $b^{\prime} \neq b$ such that $v_{2} b^{\prime}$ is a bridge of $G^{\prime}$ and $N_{G}\left(b^{\prime}\right)=\left\{v_{1}, v_{2}\right\}$. Hence $\left\{b, b^{\prime}\right\}$ is a non trivial non-dominating module of $G$ and $\left|N\left(\left\{b, b^{\prime}\right\}\right)\right|<\left|N\left(\left\{v_{1}, v_{2}\right\}\right)\right|$, a contradiction.

Consider now the line $\overline{v_{1} v_{2}}$. We claim that $\overline{v_{1} v_{2}} \notin \mathcal{L}^{\prime} \cup\left\{\overline{v_{1} t}\right\}$ which gives the result by (2). If $v_{1} v_{2}$ is an edge of $G$, then $\overline{v_{1} v_{2}}=\left\{v_{1}, v_{2}\right\}$ and the result holds. Hence we may assume that $v_{1} v_{2}$ is not an edge and thus $\overline{v_{1} v_{2}}=M \cup N(M)$. So $\overline{v_{1} v_{2}} \neq \overline{v_{1} t}$ and we may assume for contradiction that $\overline{v_{1} v_{2}} \in \mathcal{L}^{\prime}$ which implies there exists $x, y \in N(M) \cup M-\left\{v_{1}\right\}$ such that $\overline{x y}=M \cup N(M)$. If $\{x, y\} \cap\left\{b, v_{2}\right\} \neq \emptyset$, then $\overline{x y}$ must contain some vertices of $V(G)-(M \cup N(M))$, so we may assume that $\{x, y\} \subseteq N(M)$, but then $b \notin \overline{x y}$.

We now consider the case where all the non-trivial modules of $G$ are dominating.

In this case, $G$ has diameter 2. It was proven in [10] that for every graph $G$ of diameter $2, G$ either has an universal line or it has at least $|V(G)|$ distinct lines. Since what we want to prove is stronger, we cannot use this result. We will need the following lemma that was already proved in 8 .

Lemma 4.2 Let $G$ be a graph of diameter two and let $x, a, b$ be three vertices of $G$ such that $\overline{x a}=\overline{x b}$. Then either $(a, b)$ is a pair of false twins and $d(x, a)=d(x, b)=1$, or $d(x, a) \neq d(x, b)$.

PROOF - Assume that $d(x, a)=d(x, b)$. If $d(x, a)=2$, then $a \notin \overline{x b}$, a contradiction, so $d(x, a)=1$. If $a$ and $b$ are adjacent, then again $a \notin \overline{x b}$, so $a$ and $b$ are not adjacent. Assume now that there exists a vertex $c$ adjacent to $a$ but not to $b$, i.e. $d(c, a)=1$ and $d(c, b)=2$. If $d(c, a)=1$, then $c \in \overline{x b}$ and $c \notin \overline{x a}$, and if $d(c, x)=2$, then $c \notin \overline{x b}$ and $c \in \overline{x a}$, a contradiction in both cases. So $(a, b)$ is a pair of false twin. 
Notice that for any non-trivial dominating module $M$, the set $N(M)$ is a module as well and $M \cup N(M)=V(G)$. Moreover, for each $u \in M$ and each $v \in N(M)$ the line $\overline{u v}$ is given by

$$
\overline{u v}=(M-N(u)) \cup(N(M)-N(v)) .
$$

We assume first that $G$ does not contain pairs of false twins. Let $M$ be a module of $G$. For $u, u^{\prime} \in M$ and $v, v^{\prime} \in N(M)$ with $\{u, v\} \neq\left\{u^{\prime}, v^{\prime}\right\}$ we have that $\overline{u v} \neq \overline{u^{\prime} v^{\prime}}$. Hence, $\ell(G) \geq|M||N(M)|$. Since $|M| \geq 2$, then the equality $|M||N(M)|=|M|+|N(M)|+(|M|-$ 1) $(\mid N(M)-1)-1$ implies that $\ell(G) \geq|G|$ when $N(M)$ is not a singleton. If $N(M)=\{x\}$, then all the lines $\overline{x v}$ are distinct, when $v$ varies over $M$. This gives us $|V(G)|-1$ distinct lines, all containing $x$. Since $M$ has no pair of false twins, it contains at least one edge $a b$, and $\overline{a b}$ is a new line since $x \notin \overline{a b}$.

Hence, we can assume that every non-trivial module $M$ is a dominating set and the graph $G$ contains pairs of false twins.

Let $\left(u_{1}, v_{1}\right),\left(u_{2}, v_{2}\right), \ldots,\left(u_{t}, v_{t}\right)$ be the pairs of false twins of $G$ and $\operatorname{set} T=\left\{u_{1}, v_{1}, \ldots, u_{t}, v_{t}\right\}$. Since $\left\{u_{i}, v_{i}\right\}$ is a non-trivial module for $i=1, \ldots, t$, it must be a dominating module and thus $N\left(u_{i}\right)=N\left(v_{i}\right)=V(G) \backslash\left\{u_{i}, v_{i}\right\}$. This implies that all vertices in $\left\{u_{1}, v_{1}, \ldots, u_{t}, v_{t}\right\}$ are pairwise distinct, i.e. $|T|=2 t$, and that $T$ induces a complete graph minus a perfect matching.

Set $U=\left\{u_{1}, \ldots, u_{t}\right\}$ and $\mathcal{L}_{U}=\left\{\overline{u_{i} u_{j}}: 1 \leq i \neq j \leq t\right\} \cup\left\{\overline{u_{1} v_{1}}\right\}$. For $1 \leq i \neq j \leq t$, we have $\overline{u_{i} u_{j}}=\left\{u_{i}, v_{i}, u_{j}, v_{j}\right\}$ and $\overline{u_{1} v_{1}}=V(G)$. So $\left|\mathcal{L}_{U}\right|=\left(\begin{array}{l}t \\ 2\end{array}\right)+1$.

Set $R=V(G) \backslash T$. We split the rest of the proof in three cases.

Case 4.1: $R$ is empty.

In this case $|V(G)|=2 t$. If $t \in\{2,3,4\}$, then $G \in\left\{C_{4}, K_{6}^{\prime}, K_{8}^{\prime}\right\}$, which is a contradiction. If $t \geq 5$, then $\ell(G) \geq\left|\mathcal{L}_{U}\right| \geq\left(\begin{array}{l}t \\ 2\end{array}\right) \geq 2 t$ and we are done.

Case 4.2: $R$ is a clique.

Set $|R|=k \geq 1$. Set $\mathcal{L}_{R}=\{\overline{x y}: x, y \in R\}$. Notice that each pair of vertices $x, y \in R$ are true twins, resulting in $\overline{x y}=\{x, y\}$ (they are uniquely determined). Now, for each $x \in R$, set $\mathcal{L}_{x U}=\left\{\overline{x u_{i}}: i=1, \ldots, t\right\}$. Observe that $\overline{x u_{i}}=\left\{x, u_{i}, v_{i}\right\}$. It follows that lines in $\cup_{x \in R} \mathcal{L}_{x U}$ are all pairwise distinct and disjoint of $\mathcal{L}_{U}$. Moreover, lines in $\cup_{x \in R} \mathcal{L}_{x U}$ are not universal (except when $|R|=1$ and $T=\left\{u_{1}, v_{1}\right\}$, but then the graph is not 2-connected). Hence, all lines in $\mathcal{L}_{U}$, $\mathcal{L}_{R}$ and $\cup_{x \in R} \mathcal{L}_{x U}$ are pairwise distinct. So if $|R|$ and $t$ are greater than 2 we have that:

$$
\ell(G) \geq\left(\begin{array}{c}
t \\
2
\end{array}\right)+\left(\begin{array}{c}
|R| \\
2
\end{array}\right)+t|R|+1 \geq 2 t+|R|=|V(G)|
$$

If $|R|=1$ and $t \geq 2, \ell(G) \geq\left(\begin{array}{l}t \\ 2\end{array}\right)+t+1$. If $t \geq 3$ this quantity is greater than $|V(G)|$. If $t=2$ then $G=W_{4}$ which is a contradiction because $W_{4} \in \mathcal{F}$. If $|R|=1$ and $t=1$, then $G$ is not 2-connected. Hence, $R$ is not a clique.

Case 4.3: $R$ is non empty and is not a clique.

There exists $x, y \in R$ such that $x y$ is not an edge. Since $(x, y)$ is not a pair of false twin, we may assume that there exists $z \in R \backslash\{x, y\}$ such that $z$ is adjacent to $y$ but not to $x$. Set $\mathcal{L}_{x}=\{\overline{x a}: a \in U$ or $a \in R \backslash\{x\}\}$.

Suppose $\mathcal{L}_{x}$ contains an universal line $\overline{x a}$. If $a \in R$, then $d(x, a)=2$ and all the other vertices in $R$ are at distance 1 , but this would imply that $(x, a)$ is a pair of false twins. Hence, $a=u_{i}$ for some $i \in\{1,2, \ldots, t\}$. Notice that $\overline{x u_{i}}=\left\{v_{i}\right\} \cup(R \backslash N(x))$. If it is universal, then $i=1, t=1$ and $N(x)=T$. But then, $R \backslash\{x\}$ is a non-trivial non-dominanting module which is a contradiction. Hence, we can assume that $\mathcal{L}_{x}$ does not contain an universal line.

Recall that all lines in $\mathcal{L}_{U}$ are included in $T$ except for the universal line. Since no line in $\mathcal{L}_{x}$ is universal, then $\mathcal{L}_{x} \cap \mathcal{L}_{U}=\emptyset$. Moreover, since $x \notin \overline{y z}, \overline{y z} \notin \mathcal{L}_{x}$ and since $\overline{y z} \cap T=\emptyset, \overline{y z} \notin \mathcal{L}_{U}$. Hence, we have that $\ell(G) \geq\left(\begin{array}{l}t \\ 2\end{array}\right)+2+\left|\mathcal{L}_{x}\right|$ if $t \geq 2$ or $\ell(G) \geq 2+\left|\mathcal{L}_{x}\right|$ if $t=1$. 
In both cases if $\left|\mathcal{L}_{x}\right| \geq|R|+t-1$ then $\ell(G) \geq 2 t+|R|=|G|$. Thus it is enough to prove that for all $a, b \in U \cup R \backslash\{x\}$, we have $\overline{x a} \neq \overline{x b}$.

Let $a, b \in U \cup R \backslash\{x\}$ and let us prove that $\overline{x a} \neq \overline{x b}$. Since $a, b \in U \cup R \backslash\{x\},(a, b)$ is not a pair of false twins, and thus, by Lemma 4.2 , we may assume that $d(x, a) \neq d(x, b)$. Without loss of generality, $d(x, a)=1$ and $d(x, b)=2$ which implies in particular that $b \in R \backslash\{x\} f$. If $a \in R \backslash\{x\}$, then $T \subseteq \overline{x b}$ and $T \cap \overline{x a}=\emptyset$. So we may assume that $a \in U$. One of the vertices $y, z$ is distinct from $b$, say $y \neq b$. We have $[x a y]$, so $y \in \overline{x a}$. But $d(x, y)=d(x, b)=2$ which implies that $y \notin \overline{x b}$.

Thus, $\ell(G) \geq|R|+2=|G|$ which proves the Theorem.

\section{References}

[1] P. Aboulker, A. Bondy, X. Chen, E. Chiniforooshan, V. Chvátal, and P. Miao, Number of lines in hypergraphs, Discrete Applied Mathematics 171 (2014), 137-140.

[2] P. Aboulker, X. Chen, G. Huzhang, R. Kapadia and C.Supko, Lines, betweenness and metric spaces. Submitted. arXiv:1412.8283 [math.CO]

[3] P. Aboulker and R. Kapadia, The Chen-Chvátal conjecture for metric spaces induced by distance-hereditary graphs, Eur. J. Comb., 43 (2015), 1-7.

[4] L. Beaudou, A. Bondy, X. Chen, E. Chiniforooshan, M. Chudnovsky, V. Chvátal, N. Fraiman, and Y. Zwols, Lines in hypergraphs, Combinatorica 33 (6) (2013), 633-654.

[5] L. Beaudou, A. Bondy, X. Chen, E. Chiniforooshan, M. Chudnovsky, V. Chvátal, N. Fraiman and Y. Zwols, A De Bruijn-Erdős Theorem for Chordal Graphs, Electr. J. Comb., 22, 1, $\mathrm{P} 1.70,2015$.

[6] X. Chen and V. Chvátal, Problems related to a de Bruijn - Erdős theorem, Discrete Applied Mathematics 156 (2008), 2101-2108.

[7] X. Chen, G. Huzhang, P. Miao, and K. Yang, Graph metric with no proper inclusion between lines, arXiv:1402.5627 [math.MG]

[8] E. Chiniforooshan and V. Chvátal, A de Bruijn - Erdős theorem and metric spaces, Discrete Mathematics \& Theoretical Computer Science, 13 (1) (2011), 67-74.

[9] V. Chvátal, Sylvester-Gallai theorem and metric betweenness. Discrete $\&$ Computational Geometry 31 (2) (2004), 175-195.

[10] V. Chvátal, A de Bruijn-Erdős theorem for 1-2 metric spaces, Czechoslovak Mathematical Journal 64 (1) (2014), 45-51.

[11] H.S.M. Coxeter, Introduction to geometry, Wiley, New-York, 1961.

[12] N. G. De Bruijn, P. Erdős, On a combinatorial problem, Indagationes Mathematicae 10 (1948), 421-423.

[13] G.A. Dirac, On rigid circuit graphs, Abh. Math. Sem. Univ. Hambourg 25 (1961), 71-76.

[14] P. Erdős, Three point collinearity, Amer. Math. Monthly 50 (1943), Problem 4065, p. 65. Solutions in Vol. 51 (1944), 169-171. 
[15] I. Kantor, B. Patkós, Towards a de Bruijn-Erdős theorem in the L1-metric, Discrete $\mathcal{E}$ Computational Geometry 49 (2013), 659-670.

[16] K. Menger, Untersuchungen über allgemeine metrik, Math. Ann. 100 (1928), 75-163. 